\title{
Effects of Sugammadex versus Neostigmine on Intraoperative Coagulation Profiles in Patients with Thyroidectomy
}

This article was published in the following Dove Press journal:

Drug Design, Development and Therapy

\section{Dizhou Zhao \\ Jieyu Fang \\ Wei Xiong \\ Jun Lin \\ Wanmei Chen \\ Chujun Wu}

Department of Anesthesia, The First Affiliated Hospital of Sun Yat-Sen University, Guangzhou, People's Republic of China
Correspondence: Jieyu Fang Department of Anesthesia, The First Affiliated Hospital of Sun Yat-Sen University, No. 56 Zhongshan Road II, Guangzhou, People's Republic of China $\mathrm{Tel}+86-13660121136$

Email fangjy@mail.sysu.edu.cn
Background: Sugammadex has been a revolutionary reversal of neuromuscular blockade. It is known to be highly efficient. However, a change in the coagulation profile is one of the most dangerous potential complications which is a concern for both surgeon and anesthetist. Bleeding may cause hypovolemic shock, hematoma, and so on. To investigate the effects of sugammadex on coagulation profiles in patients with thyroidectomy, we compared patients that were treated with either sugammadex or neostigmine.

Patients and Methods: Eighty patients with thyroid neoplasms undergoing thyroidectomy were randomly allocated to sugammadex group (group S) or neostigmine group (group N). Induction of anesthesia was preformed using propofol, sufentanil, and rocuronium. Group $\mathrm{S}$ received sugammadex $2.0 \mathrm{mg} / \mathrm{kg}$ after trachea intubation, similarly Group $\mathrm{N}$ received neostigmine $40 \mu \mathrm{g} / \mathrm{kg}$, for reversal of rocuronium-induced neuromuscular blockade. The intraoperative coagulation profiles were monitored after the rocuronium injection (T0), 10 minutes after reversal (T1) and 30 minutes after reversal (T2) by testing activated partial thromboplastin time (APTT), prothrombin time (PT), fibrinogen (FIB), thrombin time (TT), and TEG-Haemonetics. Amount of bleeding was recorded during perioperative period.

Results: There was no significant difference in the thromboelastogram, APTT, PT, FIB, or TT measurements at each time point in Group N. The reaction time (R time) and kinetics time ( $\mathrm{K}$ time) of Group S in T1 were significantly longer than the corresponding times at T0 and $\mathrm{T} 2$, and the $\mathrm{R}$ times were significantly longer than those in Group $\mathrm{N}$ at the same time points $(\mathrm{P}<0.05)$. Additionally, in Group $\mathrm{S}$, the APTT was prolonged in $\mathrm{T} 1$ and returned to normal in $\mathrm{T} 2$.

Conclusion: The result showed that sugammadex provided transient efficacy in prolonging the coagulation parameters, while neostigmine did not change the coagulation profile.

Keywords: sugammadex, neostigmine, TEG-Haemonetics, coagulation, thyroidectomy

\section{Introduction}

Maintaining a neuromuscular blockade is an important part of general anesthesia for the purpose of providing clear surgical field, ensuring endotracheal intubation or facilitating other surgical operations. ${ }^{1,2}$ Nowadays, some operations require a change in the level of intraoperative neuromuscular blockade; that is to say, it might be essential to reverse the neuromuscular blockade rapidly. ${ }^{3}$ In the past, an anticholinesterase drug, neostigmine, was chosen to achieve the reversal, even though it could lead to complications, and the speed of reversal was unsatisfactory. ${ }^{4}$ Complications related to neostigmine include increasing gland secretion, decreasing 
heart rate, and so on. Recently, sugammadex has achieved a revolution in neuromuscular blockade reversal. Sugammadex is known to have a rapid onset, and it is highly efficient. Currently, the use of sugammadex has increased.

In thyroidectomy, the electromyographic signal endotracheal tube (EMG Endotracheal Tube) has become popular, since it assists surgeons in locating the recurrent laryngeal nerve and then avoiding nervous damage by mistake. ${ }^{5}$ Applying sugammadex after anesthesia induction reverses muscle relaxant quickly, which ensures the effect of the EMG Endotracheal Tube. ${ }^{6}$ Nevertheless, a previous in vitro study reported that sugammadex might be associated with moderate hypocoagulation in whole blood of healthy subjects. ${ }^{7}$ Perioperative bleeding is one of the most dangerous complications in patients undergoing a thyroidectomy; thus, it is a serious concern for surgeons and anesthetists. Postoperative bleeding may cause dyspnea by obstruction of airway. Therefore, in the present study sugammadex or neostigmine was applied to patients to investigate the effects of sugammadex on coagulation profiles in patients undergoing a thyroidectomy. We aimed to use qLabs Electrometer (to test activated partial thromboplastin time [APTT], prothrombin time [PT], fibrinogen [FIB], and thrombin time [TT]) and TEG-Haemonetics to assess the real-time coagulation profiles change from the patients.

\section{Methods}

Our experimental protocol which was conducted in accordance with the Declaration of Helsinki was approved by Ethics Committee of the First Affiliated Hospital of Sun yat-sen University (number: [2019]153), and it was registered in China clinical trial registry (number: ChiCTR2000030083). All of the patients signed informed consent. Study inclusion criteria were: 1) patients with American Society of Anesthesiologists (ASA) physical status ASA I to ASA II; 2) age 18-65 years; and 3) scheduled for thyroidectomy. Study exclusion criteria were: patients with cardiac disease, hepatic failure, distant metastasis of a malignant tumor, and allergy to the test drugs. Eighty patients were included in the study.

For anesthesia induction, both groups of patients were administered propofol by target-controlled infusion of 4.0 $\mu \mathrm{g} / \mathrm{mL}$, sufentanil $0.5 \mu \mathrm{g} / \mathrm{kg}$ iv, and rocuronium $0.6 \mathrm{mg} / \mathrm{kg}$ iv. After tracheal intubation, anesthesia was maintained with propofol target-controlled infusion of $2.0 \mathrm{ng} / \mathrm{mL}$ and remifentanil target-controlled infusion as $5.0 \mathrm{ng} / \mathrm{mL}$. The time at which we administered rocuronium for the neuromuscular blockade was defined as T0.
Patients in the Group $\mathrm{S}$ received sugammadex $2.0 \mathrm{mg} /$ $\mathrm{kg}$ for reversal of rocuronium-induced neuromuscular blockade. The time 10 minutes and 30 minutes after the reversal were defined as $\mathrm{T} 1$ and $\mathrm{T} 2$. The Group $\mathrm{N}$ received neostigmine $40 \mathrm{ug} / \mathrm{kg}$ for the reversal. Heart rate, blood pressure, and oxygen saturation (pulse oximeter) were monitored. If the heart rate declined to less than 50 beats per minute, a dose of $0.3 \mathrm{mg}$ atropine was administered.

We collected $3 \mathrm{~mL}$ of peripheral venous blood sample from each patient at T0 and at $10 \mathrm{~min}$ (T1) and $30 \mathrm{~min}$ (T2) after the reversal. QLabs Electrometer (Micropoint Biotechnologies, Guangdong, China) was used to test the samples for four coagulation items including prothrombin time (PT), activated partial thromboplastin time (aPTT), Thrombin time (TT), and fibrinogen (FIB) and TEGHaemonetics (thromboelastograph coagulation analyzer Model 5000, Haemoscope corporation, Boston, MA, USA) was used to test the other bleed samples for Reaction time ( $\mathrm{R}$ time), the kinetics time ( $\mathrm{K}$ time), $\alpha$ angle, and maximum amplitude (MA). $20 \mu \mathrm{g} \mathrm{CaCl}_{2}$ and the test reagent were mixed with the blood samples in the the TEG-Haemonetics cup. After 30 minutes we received the TEG items values.

The amount of blood loss of surgery was recorded. The episodes of bradycardia were recorded. The tracheal tube was extubated when the patient woke up after the surgery.

\section{Statistical Analysis}

Statistical analysis was performed with SPSS 19.0 for Windows (IBM Corporation, Armonk, NY, USA). Continuous variables were presented as mean \pm standard deviation (SD), and categorical variables were presented as frequency. Serial changes in coagulation profiles were analyzed with a repeated measures analysis of variance. Pairwise difference in coagulation profiles were analyzed with LSD$t$-test. P-values $<0.05$ were considered to be statistically significant.

\section{Results}

In this study, 80 patients undergoing a thyroidectomy had been enrolled. Baseline characteristics are presented in Table 1. There was no significant difference in age, sex, body weight, or other general characteristics between the two groups (Table 1).

\section{Coagulation Items}

There was no statistically significant difference in the APTT, PT, TT, or FIB in each time of Group N. The APTT indexes in group $\mathrm{S}$ measured at $\mathrm{T} 1$ were significantly higher than 
Table I Baseline Characteristics of Two Groups

\begin{tabular}{|c|c|c|c|c|}
\hline Characteristics & $\begin{array}{l}\text { Group } \\
S(n=40)\end{array}$ & $\begin{array}{l}\text { Group } \\
N(n=40)\end{array}$ & $x^{2} / t$ & $\mathbf{P}$ \\
\hline \multicolumn{5}{|l|}{ Sex } \\
\hline Male & 24 & 27 & & \\
\hline Female & 16 & 13 & 0.491 & 0.485 \\
\hline Age & $42.17 \pm 4.78$ & $44.38 \pm 6.85$ & 1.673 & 0.099 \\
\hline BMI $\left(\mathrm{kg} / \mathrm{m}^{2}\right)$ & $22.75 \pm 4.77$ & $22.58 \pm 4.14$ & 0.170 & 0.865 \\
\hline \multicolumn{5}{|l|}{ ASA status } \\
\hline 1 & 27 & 25 & & \\
\hline ॥ & 13 & 15 & 0.224 & 0.639 \\
\hline
\end{tabular}

that measured at T0 and T2. The T1 and T3 values were in the normal range for APTT. However, the APTT in group $\mathrm{S}$ at $\mathrm{T} 1$ was significantly higher than the $\mathrm{R}$ time in group $\mathrm{N}$ at the same time, and the results were statistically significant $(\mathrm{P}<0.05$; Table 2, Figure 1).

\section{Thromboelastogram Items}

There was no statistically significant difference in thromboelastogram indexes in each time of group $\mathrm{N}$. The $\mathrm{R}$ time in group $\mathrm{S}$ measured at $\mathrm{T} 1$ was significantly higher than that measured at $\mathrm{T} 0$ and $\mathrm{T} 2$. The $\mathrm{T} 1$ and $\mathrm{T} 3$ values were in the normal range for $\mathrm{R}$ times. However, the $\mathrm{R}$ time in group $\mathrm{S}$ at
T1 was significantly higher than the $\mathrm{R}$ time in group $\mathrm{N}$ at the same time $(\mathrm{P}<0.05)$. The $\mathrm{K}$ time in group $\mathrm{S}$ measured at $\mathrm{T} 1$ was significantly higher than that at T0. But the $\mathrm{K}$ times in group $\mathrm{S}$ at each time point were not significantly different from those in group N. There were no significant differences in the indexes of $\alpha$ angle or the MA in both groups at each time point (Table 3, Figures 2 and 3 ).

\section{Bleeding and Other Adverse Events}

There was no significant difference in blood loss between the groups. No postoperative hemorrhage was recorded in either group. During the reversal, 14 patients in group $\mathrm{N}$ experienced a decline in heart rate decrease. Among these, 10 patients received atropine $0.3 \mathrm{mg}$ then heart rate increase over 50 beats per minute. The other four patients soon recovered spontaneously. Two patients in group $\mathrm{N}$ suffered heart rate decrease and recovered spontaneously.

\section{Discussion}

In this study, the effects of sugammadex were compared versus neostigmine on intraoperative coagulation profiles in patients that underwent a thyroidectomy. The results showed that a dose of $2.0 \mathrm{mg} / \mathrm{kg}$ sugammadex might prolong the APTT index and the $\mathrm{R}$ time, and $\mathrm{K}$ time in the thromboelastograph TEG test temporarily. While a dose of

Table 2 Coagulation Items of Two Groups

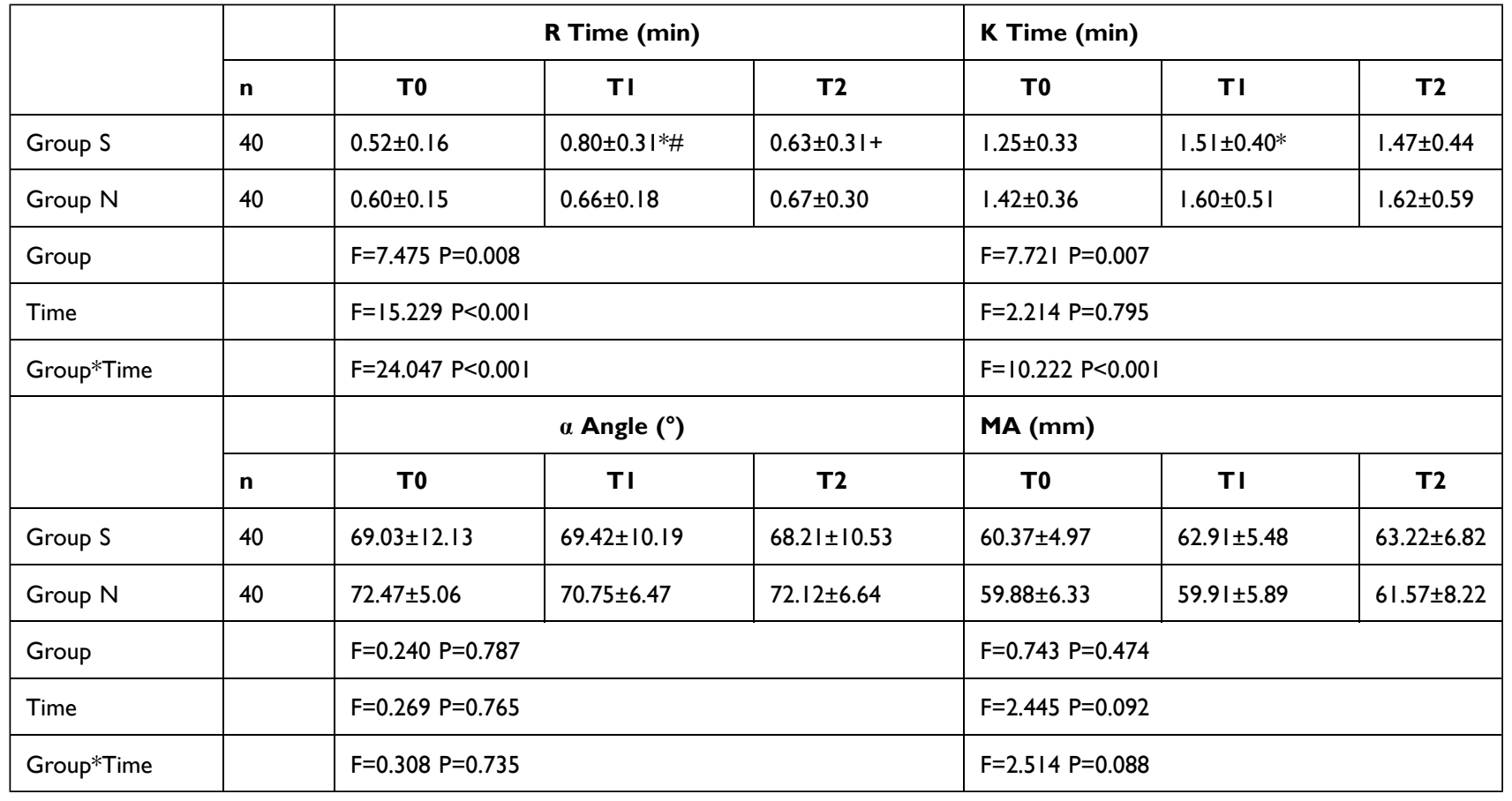

Notes: *Means compared with T0 time, it has significant difference ( $\mathrm{R}$ time: $\mathrm{LSD}-\mathrm{t}=4.647, \mathrm{P}<0.00 \mathrm{I} ; \mathrm{K}$ time LSD-t $\mathrm{t}=4.647, \mathrm{P}<0.00 \mathrm{I})$; ${ }^{+}$Means compared with $\mathrm{TI}$ time, it has significant difference (LSD-t=2.8216, $P=0.006)$ : ${ }^{\#}$ Means compared with group $N$, it has significant difference $(t=2.470, P=0.016)$. 


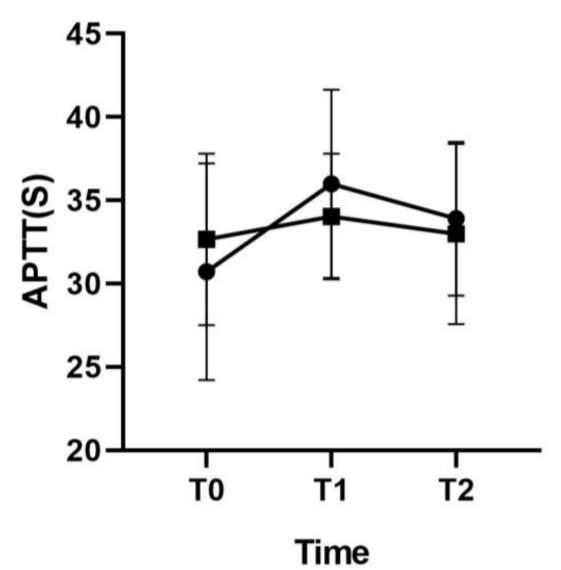

Figure I Mean change in APTT $( \pm S E)$ in two groups at different moments. T0: after rocuronium injection, $\mathrm{TI}$ : 10 minutes after reversal, T2: 30 minutes after reversal. Abbreviation: APTT, activated partial thromboplastin time.

neostigmine used to reverse neuromuscular block did not affect the coagulation profile.

Non-depolarizing neuromuscular blocking agents (NMBAs) are widely used in anesthesia induction. Previously, for reversal, neostigmine used to be chosen to weaken the activity of acetylcholinesterase. ${ }^{8}$ However, application of neostigmine may be limited by the risk that excess acetylcholine might lead to complications, such as

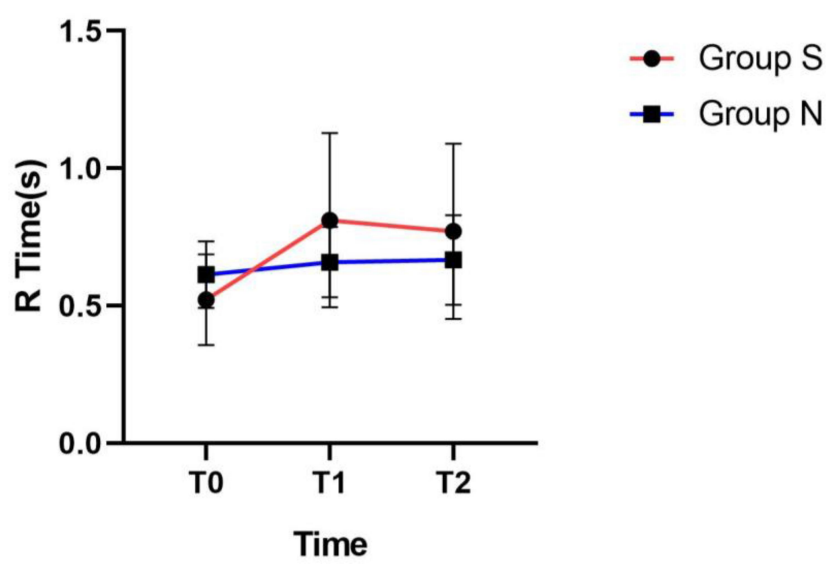

Figure 2 Mean change in $\mathrm{R}$ Time $( \pm S E)$ in two groups at different moments. T0: after rocuronium injection, TI: 10 minutes after reversal, T2: 30 minutes after reversal.

Abbreviation: $\mathrm{R}$ time, reaction time.

a decline in the heart rate and an increase in gland secretion. ${ }^{9}$

Sugammadex is one of the recent neuromuscular blocking antagonist. Sugammadex is a synthetic $\gamma$ cyclodextrin derivative, and the structure includes hydrophilic and lipophilic surfaces on different sides. ${ }^{10,11}$ This new drug works differently from neostigmine by binding up the NMBAs by the lipophilic inner cavity to compose

Table 3 Thromboelastogram Items of Two Groups

\begin{tabular}{|c|c|c|c|c|c|c|c|}
\hline & & \multicolumn{3}{|c|}{ APTT (s) } & \multicolumn{3}{|c|}{ PT (s) } \\
\hline & $\mathbf{n}$ & To & TI & T2 & TO & TI & T2 \\
\hline Group S & 40 & $30.72 \pm 6.19$ & $35.99 \pm 5.37 * \#$ & $33.80 \pm 4.39+$ & $11.93 \pm 1.32$ & $12.58 \pm 1.63$ & $|2.28 \pm 1.4|$ \\
\hline Group N & 40 & $32.66 \pm 4.89$ & $34.03 \pm 3.50$ & $32.86 \pm 5.24$ & $11.29 \pm 1.38$ & $|I .5| \pm 0.94$ & $11.21 \pm 1.67$ \\
\hline Group & & \multicolumn{3}{|c|}{$F=4.452 \quad P<0.001$} & \multicolumn{3}{|c|}{$F=2.57 \mid \mathrm{P}=0.090$} \\
\hline Time & & \multicolumn{3}{|c|}{$F=8.757 P<0.00 I$} & \multicolumn{3}{|c|}{$F=0.945 P=0.352$} \\
\hline \multirow[t]{3}{*}{ Group*Time } & & \multicolumn{3}{|c|}{$F=10.403 \quad P=0.015$} & \multicolumn{3}{|c|}{$F=0.602 P=0.469$} \\
\hline & & \multicolumn{3}{|c|}{ FIB (g/L) } & \multicolumn{3}{|c|}{ TT (s) } \\
\hline & $\mathbf{n}$ & TO & TI & T2 & TO & TI & T2 \\
\hline Group S & 40 & $2.26 \pm 0.58$ & $2.09 \pm 0.43$ & $2.26 \pm 0.47$ & $16.12 \pm 2.80$ & $15.45 \pm 2.78$ & $15.02 \pm 2.47$ \\
\hline Group N & 40 & $\mid .82 \pm 0.4 \mathrm{I}$ & $1.91 \pm 0.49$ & $|.7| \pm 0.53$ & $14.40 \pm 2.83$ & $|5.3| \pm 2.25$ & $15.12 \pm 2.27$ \\
\hline Group & & \multicolumn{3}{|c|}{$F=0.579 P=0.459$} & \multicolumn{3}{|c|}{$F=0.014 P=0.986$} \\
\hline Time & & \multicolumn{3}{|c|}{$F=0.914 P=0.350$} & \multicolumn{3}{|c|}{$F=0.757 P=0.476$} \\
\hline Group*Time & & \multicolumn{3}{|c|}{$F=0.972 P=0.388$} & \multicolumn{3}{|c|}{$\mathrm{F}=0.774 \mathrm{P}=0.453$} \\
\hline
\end{tabular}

Notes: *Means compared with T0 time, it has significant difference (LSD-t=4.39I, $\mathrm{P}<0.001$ ); ${ }^{+}$Means compared with $\mathrm{TI}$ time, it has significant difference (LSD-t=2.567, $P=0.015)$ : " Means compared with group $N$, it has significant difference $(t=2.04 I, P=0.045)$.

Abbreviations: PT, prothrombin time; aPTT, activated partial thromboplastin time; TT, thrombin time; FIB, fibrinogen. 


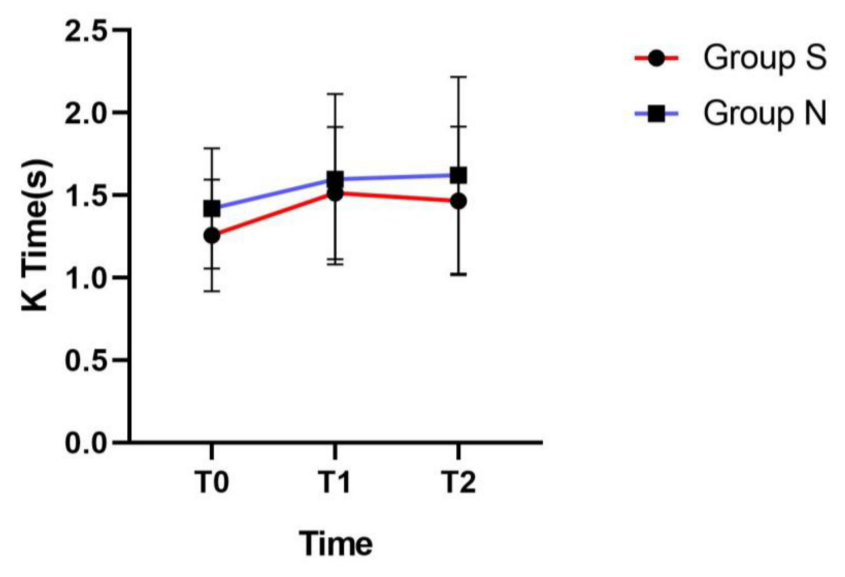

Figure 3 Mean change in $\mathrm{K}$ Time $( \pm \mathrm{SE})$ in two groups at different moments. T0: after rocuronium injection, TI: 10 minutes after reversal, T2: 30 minutes after reversal.

Abbreviation: $\mathrm{K}$ time, kinetics time.

chelate structure. $^{12}$ Thus, NMBAs are inactivated. A previous study in vitro showed that sugammadex could prolong the APTT and PT. ${ }^{13}$

In fact, the effect of coagulation in sugammadex had different results in previous pharmacological research. A study by Carron et al showed that sugammadex produced a slight effect on coagulation in morbidly obese patients, without increasing the risk of postoperative bleeding. ${ }^{14}$ Additionally, Bosch et al reported that with sugammadex $16 \mathrm{mg} / \mathrm{kg}$ in surgical patients, anti-coagulant activity would remain below thresholds observed following treatment with standard anticoagulant therapy; then, the activity decrease rapidly with declining plasma sugammadex concentrations. ${ }^{15}$

Patients that undergo a thyroidectomy typically require monitoring of the recurrent laryngeal nerve, which is aimed to make the surgical field clear. The technology of endotracheal tube really reduce the possibility of recurrent laryngeal nerve injury and helps avoid complications; ${ }^{16}$ thus, anesthetists need to reduce the dose of NMBAs or prepare to reverse neuromuscular block rapidly. ${ }^{17}$ Because of the special anatomic structure of thyroid, patients that undergo a thyroidectomy are at a higher risk of bleeding than other superficial operations. Meanwhile, acute bleeding probably cause neck hematoma and then lead to tracheal obstruction which lead to deadly dyspnea. Whether sugammadex can influence the coagulation profile, its use in patients undergoing a thyroidectomy remains controversial.

qLabs Electrometer is a machine which is able to detect coagulation items quickly by only a few drops of blood in 3 minutes. It is a point-of-care equipment, which can check coagulation items in operation room. APTT of 40 patients in group S showed a tendency to increase from $30.72 \pm 6.19 \mathrm{~s}$ to $35.99 \pm 5.37 \mathrm{~s}$ within 10 minutes; then, it came to $33.80 \pm 4.39 \mathrm{~s}$ within $30 \mathrm{~min}$ utes time after sugammadex administration. Other items did not change. APTT represent the function of the endogenous coagulation system. The prolongation might result from decreasing levels of plasma factor VIII, factor IX, and factor XI.

Furthermore, four coagulation parameters are insufficient to describe the complete coagulation process from platelet aggregation to fibrin clot formation. TEGHaemonetics is supposed to describe the coagulation process more clearly. It analyzes the function of coagulation factors, fibrin, platelets and other factors in the process of coagulation. Samples in Group S and Group N got a different result in TEG $\mathrm{R}$ time and $\mathrm{K}$ time. As we know, $\mathrm{R}$ time emerge the incubation period before fibrin formation represent the procession of initial coagulation. It relates to the function of plasma factors. $\mathrm{K}$ time shows the strength of the fibrin block and it relates to the platelet function.

In this research, both rapid coagulation parameters and the thromboelastography indexes suggested that sugammadex might act on the function of plasma factors and the platelet to prolong coagulation profiles temporarily, which is consistent with previous reports. We assume that the distinctive structure of sugammadex might play a decisive role. When sugammadex binding up the NMBAs, some chelate with moderation effect have been produced. These chelated structures might have work on the plasma coagulation factors and the platelet as well. When the drug is metabolized, this moderation effect disappears, which result in a return of coagulation factors to normal levels. This moderation effect is so slight that it does not increase intraoperative blood loss.

However, this study only focused on the effects of patients with normal coagulation function before operation in a clinical dose of sugammadex. The effects of sugammadex on patients with abnormal coagulation function before operation, especially patients with coagulation dysfunction, needs further study.

\section{Conclusion}

In this study, sugammadex is associated with transient efficacy of prolongation of coagulation parameters after administration. The changes of coagulation are so slight that it hardly increases the risk of bleeding. However, the change of 
coagulation profiles return to normal after about thirty minutes. Furthermore, neostigmine does not change in coagulation profiles.

\section{Abbreviations}

APTT, activated partial thromboplastin time; PT, prothrombin time; FIB, fibrinogen; TT, thrombin time; TEG, thromboelastogram; $\mathrm{R}$ time, reaction time; $\mathrm{K}$ time, kinetics time; $\mathrm{EMG}$, electromyographic; MA, the maximum amplitude; NMBAs, non-depolarizing neuromuscular blocking agents.

\section{Data Sharing Statement}

We intend to share all of the participant data on the ResMan public platform (website: http://www.medres man.org.cn/login.aspx). These data are available since October 2020.

\section{Disclosure}

There is no competing interest between authors and other individuality and organization.

\section{References}

1. Fuchs-Buder T, Schmartz D, Baumann C, et al. Deep neuromuscular blockade improves surgical conditions during gastric bypass surgery for morbid obesity: A randomised controlled trial. Eur J Anaesthesiol. 2019;36:486-493. doi:10.1097/EJA.0000000000000996

2. Kamen V, Vlassakov I, et al. A quest to increase safety of anesthetics by advancements in anesthesia monitoring: scientometric analysis. Drug design. Development Therapy. 2015;9:2599-2608.

3. Kim NY, Koh JC, Lee KY, et al. Influence of reversal of neuromuscular blockade with sugammadex or neostigmine on postoperative quality of recovery following a single bolus dose of rocuronium: A prospective, randomized, double-blinded, controlled study. J Clin Anesth. 2019;57:97-102. doi:10.1016/j.jclinane.2019.02.014

4. Gaver RS, Brenn BR, Gartley A, et al. Retrospective analysis of the safety and efficacy of sugammadex versus neostigmine for the reversal of neuromuscular blockade in children. Anesth Analg. 2019;129(4):1. doi:10.1213/ANE.0000000000004207

5. Moritz A, Schmidt J, Schreiner W, et al. Combined recurrent laryngeal nerve monitoring and one-lung ventilation using the EZ-Blocker and an electromyographic endotracheal tube. $J$ Cardiothorac Surg. 2019;14(1):1-8. doi:10.1186/s13019-019-0927-6
6. Schepens T, Cammu G, Saldien V, et al. Electromyographic activity of the diaphragm during neostigmine or sugammadex-enhanced recovery after neuromuscular blockade with rocuronium: A study in anaesthetised healthy volunteers. Eur J Anaesthesiol. 2015;32 (1):010201. doi:10.1097/EJA.0000000000000140

7. Kam PJD, Grobara P, Prohn M, et al. Effects of sugammadex on activated partial thromboplastin time and prothrombin time in healthy subjects. Int J Clin Pharmacol Ther. 2014;52(3):227-236. doi:10.54 14/CP201976

8. Bailey J, Wilson M, Akuffo N, et al. Evaluation of sugammadex vs neostigmine in the reversal of neuromuscular blockade. Chest $J$. 2018;154(4):890. doi:10.1016/j.chest.2018.08.799

9. Kent NB, S S L, Phillips S, et al. Therapeutic doses of neostigmine, depolarising neuromuscular blockade and muscle weakness in awake volunteers: a double-blind, placebo-controlled, randomised volunteer study. Anaesthesia. 2018;73(9):1079-1089. doi:10.1111/anae.14386

10. Nguyen-Lee J, Moreland N, Sadoughi A, et al. Sugammadex: clinical Pharmacokinetics and Pharmacodynamics. Curr Anesthesiol Rep. 2018;4(8):168-177. doi:10.1007/s40140-018-0266-5

11. Welliver M, McDonough J, Kalynych N, Redfern R. Discovery, development, and clinical application of sugammadex sodium, a selective relaxant binding agent. Drug Des Devel Ther. 2009;6 (2):49-59.

12. Abad-Gurumeta A. Evidence of residual neuromuscular block with sugammadex vs neostigmine. Br J Anaesth. 2018;120(3):603. doi:10. 1016/j.bja.2017.12.020

13. Ok LI, Sung KY, Wone CH, et al. In vitro investigation of the effects of exogenous sugammadex on coagulation in orthopedic surgical patients. BMC Anesthesiol. 2018;18(1):56-62. doi:10.1186/s12871018-0519-3

14. Carron M, Bertini D, Prandini T, et al. Effect of sugammadex on coagulation as detected by rotational thromboelastometry in morbidly obese patients. Minerva Anestesiol. 2017;84(2):178-188. doi:10.23 736/S0375-9393.17.11856-0

15. Rolien B, van Lierop M-J, de Kam P-J. A PK-PD model-based assessment of sugammadex effects on coagulation parameters. European J Pharmaceutical Sci. 2016;84:9-17. doi:10.1016/j.ejps. 2015.12.028

16. Calò PG, Medas F, Conzo G, et al. Intraoperative neuromonitoring in thyroid surgery: is the two-staged thyroidectomy justified? Int J Surg. 2017;41(Suppl 1):S13-S20. doi:10.1016/j.jjsu.2017.02.001

17. Chen HK, Chen CL, Wen KS, et al. Application of transoral continuous intraoperative neuromonitoring in natural orifice transluminal endoscopic surgery for thyroid disease: a preliminary study. Surg Endosc. 2018;32(1):517-525. doi:10.1007/s00464-017-5656-0

\section{Publish your work in this journal}

Drug Design, Development and Therapy is an international, peerreviewed open-access journal that spans the spectrum of drug design and development through to clinical applications. Clinical outcomes, patient safety, and programs for the development and effective, safe, and sustained use of medicines are a feature of the journal, which has also been accepted for indexing on PubMed Central. The manuscript management system is completely online and includes a very quick and fair peer-review system, which is all easy to use. Visit http://www. dovepress.com/testimonials.php to read real quotes from published authors. 